\title{
A Study on Non-Newtonian Transport Phenomena in Mhd Fluid Flow From a Vertical Cone With Navier Slip and Convective Heating
}

https://doi.org/10.1515/nleng-2018-0065

Received April 1, 2018; revised July 31, 2018; accepted September 30, 2018.

\begin{abstract}
In the current study is to examine numerically the effects of presence of a radial magnetic field, slip and jump conditions on the steady two-dimensional free convective boundary layer flow over an external surface of a vertical cone for an electro-conductive polymer. A proper non-similarity transformation simplifies the system of partial differential equations into a system of ordinary differential equations. The collocation formula in the MATLAB software then solves the system of non-similarity equations. The finding results show that, a weak elevation in temperature is accompanied with the increase in the Carreau fluid parameter, whereas a significant acceleration in the flow is computed near the cone surface. The study is relevant to smart coating transport phenomena.
\end{abstract}

Keywords: Magnetohydrodynamics, Carreau fluid, Thermal convection, Keller-Box method, Navier slip, Convective heating

\section{Introduction}

A good recognition gained by the Non-Newtonian fluid in the hydraulic machinery helps researchers and engineers

\footnotetext{
*Corresponding Author: CH. Amanulla, Department of Mathematics, Jawaharlal Nehru Technological University, Anantapur, Anantapuramu-515002, India E-mail: amanullamsc@gmail.com

Syed Fazuruddin, Department of Mathematics, Sreenivasa Institute of Technology and Management Studies, Chittoor-517001, India Abderrahim Wakif, Zoubair Boulahia, Hassan II University, Faculty of Sciences Aïn Chock, Laboratory of Mechanics, B.P.5366 Mâarif, Casablanca, Morocco

S. Noor Mohammed, Department of Mathematics and Computer Science, Sanskrithi School of Engineering, Puttaparthi-515134, India
}

to improve the work productivity in various fields such as drive engineering, automotive, construction, agricultural, mining, petrochemical industries, and power plant construction. For instance, the usage of the non-Newtonian lubricant in the bearings maximizes its effectiveness in the process pumps. A few salt arrangements and liquid polymers are non-Newtonian liquids, as are numerous different fluids experienced in science and innovation, for example, dental creams, physiological liquids, cleansers and paints. In a non-Newtonian liquid, the connection between the shear stretch and the shear rate is by and large non-direct and can even be time subordinate. The Carreau model although simple is useful in simulating a number of polymers, Coating hydrodynamics has been an area of considerable interest, since Landau and Levich [1] have published in their pioneering work in 1942, in which an elegant formulation was developed for the thickness of the Newtonian viscous fluid films, which is deposited on a plate withdrawn vertically from a bath at a constant velocity. Lawrence and Zhou [2] have reported in their interesting investigation that the non-Newtonian polymer fluids are encountered in many modern industries, especially in the polymer coating processes. Numerous researchers have investigated the coating dynamics of different geometrical bodies (plates, cones, spheres, cylinders) with non-Newtonian liquids and have employed a range of mathematical constitutive equations. Jenekhe and Schuldt [3] have studied the coating flows of powerlaw and Carreau fluids on spinning disks. Campanella et al. [4] have treated the dip coating of a circular cylinder in non-Newtonian power-law fluids. Zevallos et al. [5] have used the finite element for simulating the forward roll coating flows for viscoelastic liquids using both Oldroyd$B$ and FENE-P models. However, these studies ignored the heat transfer which may be critical in certain coating systems. This remark has been confirmed by Mitsoulis [6] and Mark [7], they have shown in their research papers that the diffusion of heat can modify the polymer properties significantly. 
For portraying the non-Newtonian stream conduct, Navier-Stokes conditions are insufficient. We require some physical models to fill this hole, for example, the Cross and Ellis display, the Carreau show, the Casson demonstrate, and so on. Non-Newtonian liquids are extremely useful in glass blowing, optimal design, paper creation, consistent throwing, and so on. The use of physical states of non-Newtonian liquid streams is directly trying for researchers, mathematicians and physicists, in viable and in addition hypothetical examinations. The explanation for this is these liquids are exceptionally perplexing in nature and there is no specific constitutive condition for speaking to all stream properties of non-Newtonian liquids. In Carreau liquids the thickness is lessened with rising shear stretch rates. This model has discovered some fame in building reenactments.

The classical Navier-Stokes theory does not describe sufficiently the flow properties of polymeric fluids, colloidal suspensions, and fluids having certain additives. Uddin et al. [8] presented similarity solutions of free convective power law non-Newtonian nanofluid flow past a convectively horizontal plate. Uddin et al. [9] pointed out the characteristics of laminar free convective slip flow of an electrically conducting Newtonian nanofluid from a shrinking sheet in a quiescent fluid. Analysis of velocity slip effects on peristalsis of copper-water nanofluid in a curved channel is reported by Hayat et al. [10]. Farooq et al. [11] used Mathematica 8 computational software for integration peristalsis of Sisko nanoliquid with gyrotactic microorganism in a curved channel. The free convection gyrotactic bio-convection flow of nanofluid along an upward facing and translating horizontal plate with Lie group analysis and Chebyshev collocation method solved by Uddin et al. [12]. Amanulla et al. [13] investigated slip effects on Williamson fluid flow from an isothermal sphere. They notice that the behavior of fluid on velocity and temperature distributions when thermal and velocity slips are considered. Nagendra et al. [14] have used the MATLAB software to analyze the Navier slip and Newtonian heating effects on the transient free convection flow of a Casson nanofluid from a truncated vertical cone with applications in nanotechnology science. The transport from external surfaces of curved bodies (e.g., vertical cones) has also stimulated some interest in recent years. Such flows are of relevance to chemical engineering systems and also materials synthesis. Tripathi and Bég [15] have analyzed the peristaltic propulsion with the thermal diffusion using the Jeffery model. Cheng [16] has also examined the natural convection heat transfer about a vertical cone embedded in a porous medium with isothermal wall conditions. Yih [17] has studied the effect of thermal radiation flux on free convection about a truncated cone. Gorla et al. [18] have investigated the micropolar convection boundary layer flow from a cone. Uddin et al. [19] reported on heat transfer of a moving permeable non-linearly extrusion stretching sheet with radiation flux model, velocity slip, thermal and mass convective boundary conditions. Johnson-Segalman fluid peristalsis flow of non-Newtonian material is investigated by Farooq et al. [20]. Hayat et al. [21] addressed magnetic field on peristaltic flow of an incompressible Carreau fluid in a curved channel with Joule heating effect. Hayat et al. [22] analyzed the influence of radiation in MHD peristalsis of Carreau Nano liquids. Saleem et al. [23, 24] presented extensive numerical solutions for vertically rotating cone of a non-Newtonian fluid with convective boundary conditions. Amanulla et al. [25] analyzed the steady free convection flow of a viscous incompressible fluid past an infinite vertical plate in the presence of velocity and thermal slip effects. Other studies on the viscoelastic fluid over vertical cone include Saleem et al. [26, 27], Amanulla et al. [28].

Among the interesting viscoelastic model in nonNewtonian fluid mechanics we find the Carreau fluid model, which is degenerated to a Newtonian fluid at a very high wall shear stress. This model approximates reasonably well the rheological behavior of a wide range of industrial liquids including biotechnological detergents, physiological suspensions, foams, geological material, cosmetics and syrups. Many researchers have explored a range of industrial and biological flow problems using the Carreau model. Khellaf and Lauriat [29] have investigated the Carreau fluid flow and heat transfer between two concentric cylinders. Khan et al. [30] have analyzed the MHD non-Newtonian Carreau fluid flow over a convective surface with nonlinear radiative heat transfer effects. Raju and Sandeep [31] have examined the heat and mass transfer in Falkner-Skan Carreau fluid flow past an isothermal wedge. Akbar et al. [32] have studied theoretically the MHD stagnation-point flow of Carreau fluid over a shrinking sheet. Reddy and Sandeep [33] have investigated the steady radiative MHD free convection flow of a Carreau fluid from a shrinking sheet with thermal and concentration slips. The Carreau fluid model quite accurately describes the characteristics of relaxation and retardation times, which arise in certain polymeric flows. Furthermore, this model utilizes time derivatives rather than converted derivatives, which facilitates numerical solutions in boundary value problems.

The previous studies invariably assumed the classical boundary conditions. However, the slip effects have shown to be important in numerous polymeric transport processes including the production stage of polymers from the 
raw materials. Black [34] has reported that the slip effect on the wall must be taken into account during converting high molecular weight products into specific products. Consequently, many researchers, primarily in chemical engineering have studied experimentally and numerically the influence of wall slip on polymer dynamics. The important works in this regard include Wang et al. [35] who considered low density polyethylene liquids, Piau et al. [36] who addressed polymer extrudates, Piau and Kissi [37] who quantified macroscopic wall slip in polymer melts, Lim and Schowalter [38] who studied boundary slip in polybutadiene flows and Hatzikiriakos and Kalogerakis [39] who studied molten polymer wall slip. The wall slip in thermal polymer processing was considered by Liu and Gehde [40], in which the slip was shown to significantly modify temperature distribution in polymers. Hatzikiriakos and Mitsoulis [41] have presented closed form solutions and finite element computations for wall slip effects on pressure drop of power law fluids in tapered dies. Many studies of the effects of both the momentum slip (i.e., velocity slip) and the thermal slip on transport phenomena have also been reported. Grubka and Bobba [42] proved that the Prandtl number and temperature parameter are able to limit the heat transfer characteristics past the continuous stretching surface and indirectly noticed that the Crane's [43] solution is actually an exact solution of the Navier-Stokes equations. Wang [44] studied the vertical free convection problem and found the closed form solutions for the temperature distribution with some selected values of the Prandtl numbers and also presented the three-dimensional similarity solution to the full NavierStokes-Boussinesq equations. Following are some of the significant literatures that analyzed the fluid flow past a stretching sheet: Gupta and Gupta [45]. Recently, the researchers [46-48] discussed the non-Newtonian fluid flow of vertical surfaces with velocity and temperature jump boundary conditions. In these studies, they found very interesting solutions as the momentum slip and thermal slip parameter has tendency to control the skin friction and local Nusselt number profiles and also the non-Newtonian fluids are regulating the temperature profiles of the flow.

Newtonian heating is however physically more realistic for materials processing operations, as studied in our paper. It captures better the wall thermal conditions in non-Newtonian sheet processing. It allows conjugate heat transfer to be simulated at the wall and avoids under prediction encountered with conventional thermal boundary conditions. Therefore, the heat transfer from the surface is taken to be proportional to the local surface temperature in Newtonian heating and this mimics quite well physically observed phenomena in thermal materials process- ing. The hot stretching wall can be better simulated than with standard thermal boundary conditions and this improves the correlation between our mathematical model and manufacturing processes involving thermal treatment of non-Newtonian liquids (polymers). Newtonian heating was introduced as a term by Merkin [49] and has been successfully employed by many authors in polymeric and magnetic material flow simulations including Ramzan et al. [50], Awais et al. [51] and Uddin et al. [52]. These studies all confirmed that very different and better results are achieved with Newtonian boundary conditions for heating compared with conventional thermal wall conditions. The present study finds applications in electro-conductive thermal polymer processing systems.

All the above studies have focused on the flow over a plane, over a parabolic leading edge parabolic or in many other regions. In this work, we investigate numerically the two-dimensional MHD Carreau fluid flow Past a Vertical cone in the presence of velocity and Convective heating effects, using the Keller-Box numerical method. The impact of different parameters on the fluid flow, thermal fields, skin friction and rate of heat transfer are shown graphically. Tabular results are presented to discuss the wall friction and the reduced Nusselt number.

\section{Formulation of the Problem}

Consider the steady laminar boundary layer flow of twodimensional incompressible Carreau fluid over an isothermal horizontal circular cylindrical body of fixed radius $a$, in the presence of a uniform radial magnetic field $\mathrm{B}_{0}$. In this investigation, the induced magnetic field, the Hall effect and the viscous and Joule dissipation terms are assumed to be negligible, so that the surface temperature of the cylinder $T_{w}$ is taken greater than the ambient temperature of the fluid $T_{\infty}$. (e.g., heated cylinder). The physical model considered is illustrated in Fig. 1.

The differential equations governing the present problem are given as:

$$
\begin{gathered}
\nabla \cdot \mathrm{V}=0 \\
\rho\left[\frac{\partial \mathrm{V}}{\partial t}+(\mathrm{V} \cdot \nabla) \mathrm{V}\right]=-\nabla P+\nabla \cdot \bar{\tau}+\rho \mathrm{g}+\sigma\left(\mathrm{V} \times \mathrm{B}_{0}\right) \times \mathrm{B}_{0} \\
\rho c\left[\frac{\partial T}{\partial t}+(\mathrm{V} \cdot \nabla) T\right]=-\nabla \cdot \mathrm{q}
\end{gathered}
$$

Here, $\mathrm{V}$ is the velocity vector, $\bar{\tau}$ is the extra stress tensor, $\mathrm{q}$ is the heat flux vector, where $\mathrm{V}=(U, V)$ and $\mathrm{q}=-k \nabla T$. 


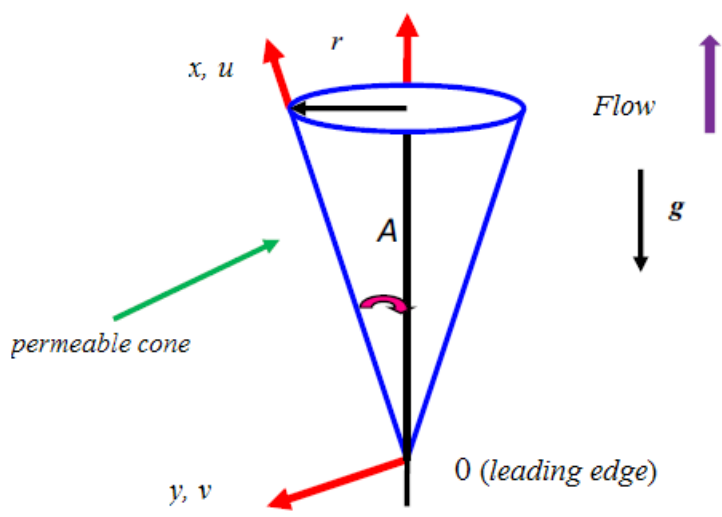

Fig. 1: Magnetohydrodynamic non-Newtonian heat transfer from a cone

In the case where the infinite shear rate viscosity $\mu_{\infty}$ of this kind of non-Newtonian fluids is neglected (i.e., $\mu_{\infty} \approx 0$ ), the extra stress tensor $\bar{\tau}$ is defined as:

$$
\bar{\tau}=\mu_{0}\left(1+(\Gamma \dot{y})^{2}\right)^{\frac{(n-1)}{2}}\left(\overline{\mathrm{L}}+\overline{\mathrm{L}}^{\mathrm{T}}\right)
$$

Here $\mu_{0}$ is the zero shear rate viscosity, $\Gamma$ is the time constant, $n$ is the power-law index , $\dot{y}$ is the shear rate and $\Pi$ is the second invariant strain rate tensor (i.e., $\left.\Pi=\operatorname{trace}\left[\left(\overline{\mathrm{L}}+\overline{\mathrm{L}}^{\mathrm{T}}\right)^{2}\right]\right)$, where $\overline{\mathrm{L}}=\nabla \mathrm{V}$ and $\dot{y}=$ $\sqrt{\frac{1}{2} \Pi \text {. }}$

We consider in the constitutive Eq. (4) the case for which $\Gamma \dot{y} \ll 1$, so we can write:

$$
\bar{\tau}=\mu_{0}\left(1+\frac{(n-1)}{2}(\Gamma \dot{y})^{2}\right)\left(\overline{\mathrm{L}}+\overline{\mathrm{L}}^{\mathrm{T}}\right)
$$

Under the Boussinesq and boundary layer approximations, the simplified steady governing equations are expressed as follows:

$$
\begin{gathered}
\frac{\partial(r U)}{\partial x}+\frac{\partial(r V)}{\partial y}=0 \\
U \frac{\partial U}{\partial x}+V \frac{\partial U}{\partial y}=g \beta\left(T-T_{\infty}\right) \cos A-\frac{\sigma B_{0}^{2}}{\rho} U+v \frac{\partial^{2} U}{\partial y^{2}} \\
+v \frac{3(n-1) \Gamma^{2}}{2}\left(\frac{\partial U}{\partial y}\right)^{2} \frac{\partial^{2} U}{\partial y^{2}} \\
U \frac{\partial T}{\partial x}+V \frac{\partial T}{\partial y}=\alpha \frac{\partial^{2} T}{\partial y^{2}}
\end{gathered}
$$

Rewriting Eqs. (6) - (8) in terms of the stream function $\psi$ (i.e., $U=\partial \psi / \partial y$ and $V=-\partial \psi / \partial x$ ) and the temperature $T$, we obtain:

$$
\begin{aligned}
& \frac{\partial \psi}{\partial y} \frac{\partial^{2} \psi}{\partial x \partial y}-\frac{\partial \psi}{\partial x} \frac{\partial^{2} \psi}{\partial^{2} y}=g \beta\left(T-T_{\infty}\right) \cos A \\
& -\frac{\sigma B_{0}^{2}}{\rho} \frac{\partial \psi}{\partial y}+v \frac{\partial^{3} \psi}{\partial y^{3}}+v \frac{3(n-1) \Gamma^{2}}{2}\left(\frac{\partial^{2} \psi}{\partial y^{2}}\right)^{2} \frac{\partial^{3} \psi}{\partial y^{3}}
\end{aligned}
$$

$$
\frac{\partial \psi}{\partial y} \frac{\partial T}{\partial x}-\frac{\partial \psi}{\partial x} \frac{\partial T}{\partial y}=\alpha \frac{\partial^{2} T}{\partial y^{2}}
$$

Considering the case of presence of velocity slip and temperature jump conditions at the cylindrical exterior wall, we can write the boundary conditions imposed to the system of Eqs. (9) and (10) as follows:

$$
\begin{gathered}
u=N_{0} \frac{\partial u}{\partial y}, v=0, T=-k \frac{\partial T}{\partial y}=h_{w}\left(T_{w}-T\right) \text { at } y=0 \\
u \rightarrow 0, v \rightarrow 0, T \rightarrow T_{\infty} \text { as } y \rightarrow \infty
\end{gathered}
$$

The parameters $N_{0}$ and $h_{w}$ used in the boundary conditions (6) represent the thermal jump and momentum slip factors, respectively. For recovering the no-slip case, one can take $N_{0}=h_{w}=0$.

In order to write the governing equations and the boundary conditions in dimensionless form, the following non-dimensional quantities are introduced:

$$
\begin{aligned}
& \xi=\frac{V_{0} x}{v G r^{1 / 4}}, \eta=\frac{y}{x}(G r)^{1 / 4}, \psi=v(G r)^{1 / 4}\left(f(\xi, \eta)+\frac{\xi}{2}\right), \\
& G r_{x}=\frac{g \beta_{T}\left(T_{w}-T_{\infty}\right) x^{3} \cos \mathrm{A}}{4 v^{2}}, \theta(\xi, \eta)=\frac{T-T_{\infty}}{T_{w}-T_{\infty}}
\end{aligned}
$$

By applying the non-similar transformations of Eq. (12) on the system of Eqs. (9) - (11), we obtain:

$$
\begin{gathered}
f^{\prime \prime \prime}(\eta)+\frac{7}{4} f(\eta) f^{\prime \prime}(\eta)+\frac{3(n-1)}{2} W e\left(f^{\prime \prime}(\eta)\right)^{2} f^{\prime \prime \prime}(\eta) \\
-\frac{1}{2}(f(\eta))^{2}+\theta-M f(\eta)=\frac{7}{4} \xi\left(f^{\prime} \frac{\partial^{2} f}{\partial \eta \partial \xi}-\frac{\partial^{2} f}{\partial \eta^{2}} \frac{\partial f}{\partial \xi}\right) \\
\operatorname{Pr}^{-1} \theta^{\prime \prime}(\eta)+\frac{7}{4} f(\eta) \theta(\eta)=\frac{7}{4} \xi\left(\frac{\partial f}{\partial \eta} \frac{\partial \theta}{\partial \xi}-\frac{\partial \theta}{\partial \eta} \frac{\partial f}{\partial \xi}\right)
\end{gathered}
$$

$$
f=0, \quad f^{\prime}(\eta)=S_{f} f^{\prime \prime}(\eta), \quad \theta=1+S_{T} \theta^{\prime}(\eta) \text { at } \eta=0
$$$$
f^{\prime} \rightarrow 0, \quad \theta \rightarrow 0 \text { as } \eta \rightarrow \infty
$$

The skin friction coefficient $C_{f}$ and the local Nusselt number $\mathrm{Nu}$ can be found using the following expressions:

$$
\begin{gathered}
\frac{1}{2} G r^{-3 / 4} \mathrm{C}_{f}=f(\xi, 0)\left(1+\frac{(n-1) W e}{2} f^{2}(\xi, 0)\right) \\
G r^{-1 / 4} \mathrm{Nu}=-\theta^{\prime}(\xi, 0)
\end{gathered}
$$

\section{Computational Solution With Keller-Box Implicit Method}

The coupled boundary layer equations in a $(\xi, \eta)$ coordinate system remain strongly nonlinear. An efficient implicit finite difference method, called the Keller-Box 
scheme, is therefore used as numerical technic to solve the boundary value problem defined by Eqs. (13) and (14) with the boundary conditions (15). This technique has been described succinctly in Cebeci and Bradshaw [54] and Keller [55]. It has been used recently in polymeric flow dynamics by Amanulla et al. [56, 57], Nagendra et al. $[58,59]$ for viscoelastic models. Very few of these papers however have provided guidance for researchers as to customization of the Keller-Box scheme to heat transfer problems. We have included the full details for discretizing the present problem with this procedure, so that the implementation of Keller's scheme involves the following steps:

1. Reduction of the $N^{\text {th }}$ order corresponding to the system of partial differential equations to a set of $N$ first order equations.

2. Discretization of the obtained first - order equations with finite difference schemes.

3. Quasilinearization of the nonlinear Keller algebraic equations.

4. Solve the system of linear Keller algebraic equations using an efficient block tridiagonal elimination procedure.

Considering the following change of variables:

$$
\begin{aligned}
& \frac{\partial f}{\partial \eta}=f^{\prime}=u \\
& \frac{\partial u}{\partial \eta}=u^{\prime}=v \\
& \frac{\partial \theta}{\partial \eta}=\theta^{\prime}=t
\end{aligned}
$$

Eqs. (13) and (14) can be reduced to the following form:

$$
\begin{gathered}
v^{\prime}+\frac{7}{4} f v+\frac{3(n-1) W e}{2} v v^{\prime}-\frac{1}{2} u^{2}+\frac{\sin (\xi)}{\xi} s-M u \\
=\frac{7}{4} \xi\left(u \frac{\partial u}{\partial \xi}-v \frac{\partial f}{\partial \xi}\right) \\
\operatorname{Pr}^{-1} t^{\prime}+\frac{7}{4} f t=\frac{7}{4} \xi\left(u \frac{\partial s}{\partial \xi}-t \frac{\partial f}{\partial \xi}\right)
\end{gathered}
$$

Here, the primes denote differentiation with respect to $\eta$ and $\theta=s$.

In terms of the dependent variables, the boundary conditions (15) become:

$$
\begin{gathered}
f=0, \quad \frac{\partial f}{\partial \eta}=S_{f}\left(\frac{\partial^{2} f}{\partial \eta^{2}}\right), \quad\left(\frac{\partial \theta}{\partial \eta}\right)=B i(\theta-1) \text { at } \eta=0 \\
f^{\prime} \rightarrow 0, \quad \theta \rightarrow 0 \text { as } \eta \rightarrow \infty
\end{gathered}
$$

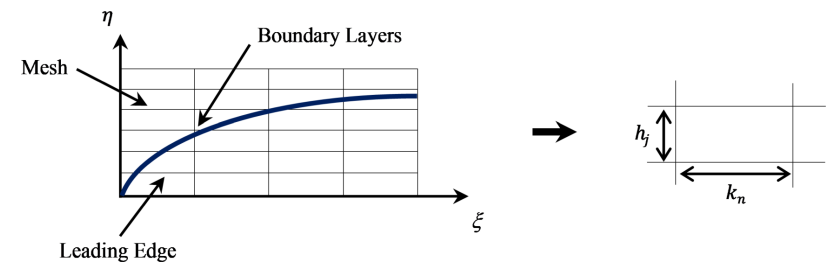

Fig. 2: Keller - Box element and boundary layer mesh

A two-dimensional computational mesh is imposed on the $\xi-\eta$ plane as shown in Fig. 2. The stepping process is defined by:

$$
\begin{gathered}
\eta_{0}=0, \quad \eta_{j}=\eta_{j-1}+h_{j}, \quad 1 \leq j \leq J, \quad \eta_{J} \equiv \eta_{\infty} \\
\xi^{0}=0, \quad \xi^{n}=\xi^{n-1}+k_{n}, \quad 1 \leq n \leq N
\end{gathered}
$$

Here, $k_{n}$ and $h_{j}$ denote the step distances in the $\xi$ and $\eta$ directions, respectively.

If $g_{j}^{n}$ denotes the value of any variable at $\left(\eta_{j}, \xi^{n}\right)$, then the variable and derivative terms appeared in Eqs. (21) and (22) at $\left(\eta_{j-1 / 2}, \xi^{n-1 / 2}\right)$ are replaced by:

$$
g_{j-1 / 2}^{n-1 / 2}=\frac{1}{4}\left(g_{j}^{n}+g_{j-1}^{n}+g_{j}^{n-1}+g_{j-1}^{n-1}\right)
$$

$$
\begin{aligned}
& \left(\frac{\partial g}{\partial \eta}\right)_{j-1 / 2}^{n-1 / 2}=\frac{1}{2 h_{j}}\left(g_{j}^{n}-g_{j-1}^{n}+g_{j}^{n-1}-g_{j-1}^{n-1}\right) \\
& \left(\frac{\partial g}{\partial \xi}\right)_{j-1 / 2}^{n-1 / 2}=\frac{1}{2 k^{n}}\left(g_{j}^{n}-g_{j-1}^{n}+g_{j}^{n-1}-g_{j-1}^{n-1}\right)
\end{aligned}
$$

The finite difference approximations at the mid-point $\left(\eta_{j-1 / 2}, \xi^{n}\right)$ for the variables $u, v$ and $t$ are given as:

$$
\begin{aligned}
& h_{j}^{-1}\left(f_{j}^{n}-f_{j-1}^{n}\right)=u_{j-1 / 2}^{n} \\
& h_{j}^{-1}\left(u_{j}^{n}-u_{j-1}^{n}\right)=v_{j-1 / 2}^{n} \\
& h_{j}^{-1}\left(s_{j}^{n}-s_{j-1}^{n}\right)=t_{j-1 / 2}^{n}
\end{aligned}
$$

Hence, the appropriate discretized form of Eqs. (21) and (22) are written as follows:

$$
\begin{aligned}
& \left(v_{j}-v_{j-1}\right)+(1+\alpha) \frac{7 h_{j}}{16}\left(f_{j}+f_{j-1}\right)\left(v_{j}+v_{j-1}\right) \\
& -\frac{(1+\alpha) h_{j}}{4}\left(u_{j}+u_{j-1}\right)^{2}+\frac{3(n-1) W e}{4}\left[\left(v_{j}\right)^{2}-\left(v_{j-1}\right)^{2}\right] \\
& +\frac{h_{j}}{2}\left(s_{j}+s_{j-1}\right)-\frac{M h_{j}}{2}\left(u_{j}+u_{j-1}\right) \\
& -\frac{7 \alpha h_{j}}{8} f_{j-1 / 2}^{n-1}\left(v_{j}+v_{j-1}\right)+\frac{7 \alpha h_{j}}{8} v_{j-1 / 2}^{n-1}\left(f_{j}+f_{j-1}\right) \\
& =\left[R_{1}\right]_{j-1 / 2}^{n-1}
\end{aligned}
$$




$$
\begin{aligned}
& \operatorname{Pr}^{-1}\left(t_{j}-t_{j-1}\right)+(1+\alpha) \frac{7 h_{j}}{16}\left[\left(f_{j}+f_{j-1}\right)\left(t_{j}+t_{j-1}\right)\right] \\
& -\frac{7 \alpha h_{j}}{16}\left[\left(u_{j}+u_{j-1}\right)\left(s_{j}+s_{j-1}\right)\right]+\frac{7 \alpha h_{j}}{8} s_{j-1 / 2}^{n-1}\left(u_{j}+u_{j-1}\right) \\
& -\frac{\alpha h_{j}}{2} u_{j-1 / 2}^{n-1}\left(s_{j}+s_{j-1}\right)-\frac{7 \alpha h_{j}}{8} f_{j-1 / 2}^{n-1}\left(t_{j}+t_{j-1}\right) \\
& +\frac{7 \alpha h_{j}}{8} t_{j-1 / 2}^{n-1}\left(f_{j}+f_{j-1}\right)=\left[R_{2}\right]_{j-1 / 2}^{n-1}
\end{aligned}
$$

Here, the following abbreviations are used:

$$
\begin{aligned}
& \quad \frac{-\left[R_{1}\right]_{j-1 / 2}^{n-1}}{h_{j}}=\left(\frac{v_{j}-v_{j-1}}{h_{j}}\right)+\frac{7}{4}(1-\alpha)\left(f_{j-1 / 2} v_{j-1 / 2}\right) \\
& +\frac{3(n-1) W e}{2}\left(v_{j-1} v_{j-1 / 2}^{\prime}\right)+\left(\frac{7 \alpha-2}{4}\right)\left(u_{j-1 / 2}\right)^{2} \\
& +s_{j-1 / 2}-M u_{j-1 / 2} \\
& \frac{-\left[R_{2}\right]_{j-1 / 2}^{n-1}}{h_{j}}=\operatorname{Pr}^{-1}\left(\frac{t_{j}-t_{j-1}}{h_{j}}\right)+\frac{7}{4}(1-\alpha)\left(f_{j-1 / 2} t_{j-1 / 2}\right) \\
& +\frac{7}{4} \alpha\left(u_{j-1 / 2} s_{j-1 / 2}\right)
\end{aligned}
$$

The system of Eqs. (32) and (33) is solved subject to the following boundary conditions:

$$
f_{0}^{n}=u_{0}^{n}=0, \theta_{0}^{n}=1, u_{J}^{n}=0, v_{J}^{n}=0, \theta_{J}^{n}=0
$$

The emerging nonlinear system of algebraic equations is linearized by means of Newton's method and then solved by the block elimination procedure. The accuracy of computations is influenced by the number of mesh points in both directions. After experimenting with various grid sizes in the $\eta$ direction a larger number of mesh points are selected whereas in the $\xi$ direction significantly less mesh points are utilized. The numerical value of $\eta_{\max }$ has been set at 10 and this defines a sufficiently large value at which the prescribed boundary conditions are satisfied, whereas the limited value $\xi_{\max }$ is set at 3 for this flow domain. Mesh independence is therefore achieved in the present computations. The present problem is solved using the Keller-Box finite difference scheme with the help of software MATLAB.

\section{Numerical Results and Interpretation}

In order to verify the accuracy of the Keller-Box solutions, computations are benchmarked with earlier results reported by Alam et al. [53] , via the values of the skin friction and heat transfer coefficients $C_{f}$ and $\mathrm{Nu}$ of a Newtonian fluid (i.e., $W e=0$ ), respectively, for various values of the
Table 1: Comparison of $C_{f}$ and $\mathrm{Nu}$ with the existing works of Alam et al. [53], in the case where $\operatorname{Pr}=1, B i \rightarrow \infty$ and $W e=S_{f}=M=$ 0 .

\begin{tabular}{ccccc}
\hline \multirow{2}{*}{$\xi$} & \multicolumn{2}{c}{$f^{\prime \prime}(\xi, 0)$} & \multicolumn{2}{c}{$-\theta^{\prime}(\xi, 0)$} \\
\cline { 2 - 5 } & $\begin{array}{c}\text { Alam et } \\
\text { al. [53] }\end{array}$ & $\begin{array}{c}\text { Present } \\
\text { Solutions }\end{array}$ & $\begin{array}{c}\text { Alam et } \\
\text { al. [53] }\end{array}$ & $\begin{array}{c}\text { Present } \\
\text { Solutions }\end{array}$ \\
\hline 0 & 0.891936 & 0.891940 & 0.420508 & 0.420502 \\
0.5 & 0.896407 & 0.896403 & 0.620748 & 0.620744 \\
1.0 & 0.856963 & 0.856965 & 0.848025 & 0.848030 \\
1.5 & 0.779210 & 0.779211 & 1.129365 & 1.129369 \\
2.0 & 0.674111 & 0.674110 & 1.441742 & 1.441747 \\
3.0 & 0.434152 & 0.434151 & 2.202662 & 2.202661 \\
\hline
\end{tabular}

parameter $\xi$, in the case where $\operatorname{Pr}=1, S_{f}=0, B i \rightarrow \infty$ and $M=0$. The results of this comparison are summarized in Table 1. Very close correlation is achieved between the Keller - Box computational results and the solutions of Alam et al. [53]. Hence, the Keller-Box numerical code used in this investigation leads to quite accurate numerical results.

The effects of major controlling parameters on the profiles of the functions $f^{\prime}(\xi, \eta), \theta(\xi, \eta), G r^{-3 / 4} C_{f}$ and $\mathrm{Gr}^{-1 / 4} \mathrm{Nu}$ are analyzed graphically through the curves plotted in Figs. 3 - 7. It is shown from Eq. (12) that there is a direct relation between the function $f^{\prime}(\xi, \eta)$ and the dimensionless velocity of the Carreau fluid. Hence, these figures allow to analyze the distributions of the dimensionless velocity, temperature, skin friction coefficient and Nusselt number.

Figs. $3-7$ exhibit the velocity and temperature distributions (i.e., $f^{\prime}(\xi, \eta)$ and $\left.\theta(\xi, \eta)\right)$ for various thermophysical parameters, namely the Carreau fluid parameter (i.e., Weissenberg number) $W e$, the magnetic body force parameter $M$, the velocity slip parameter $S_{f}$, the Biot number $B i$, the Prandtl numberPr , the power law indexn and the steam wise coordinate $\xi$.

Fig. 3 illustrates the influence of the Weissenberg number $W e$ on the velocity and temperature distributions. It is shown from Fig. 3(a) that the dimensionless velocity component is considerably reduced with the increase in the value of the parameter We. This result can be explained by the increase in the relaxation time of the polymer fluid, which creates a resistance to the fluid flow. The Weissenberg number We introduces a kind of nonlinearity to the system. Furthermore, it is noted that the Weissenberg number $W e$ is raised in connection with some higher order derivatives in the momentum boundary layer equation, especially in the mixed derivative $W e\left(\partial^{2} f / \partial \eta^{2}\right)^{2}\left(\partial^{3} f / \partial \eta^{3}\right)$. From Fig. 3(b), it is quite clear that an increase in the Weissenberg number $W e$ enhances 


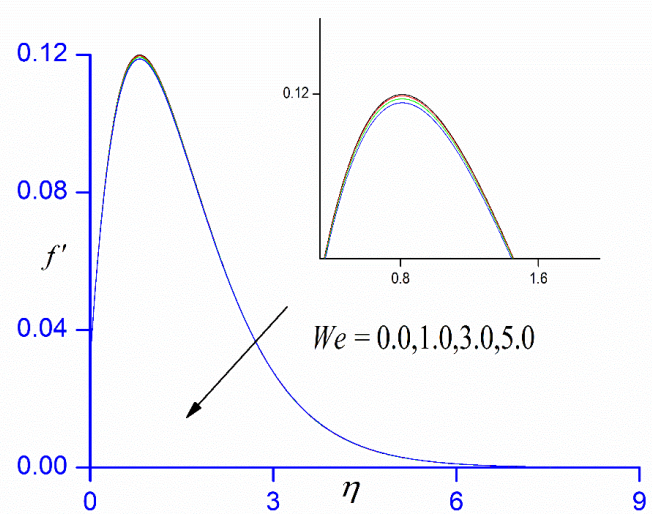

(a)

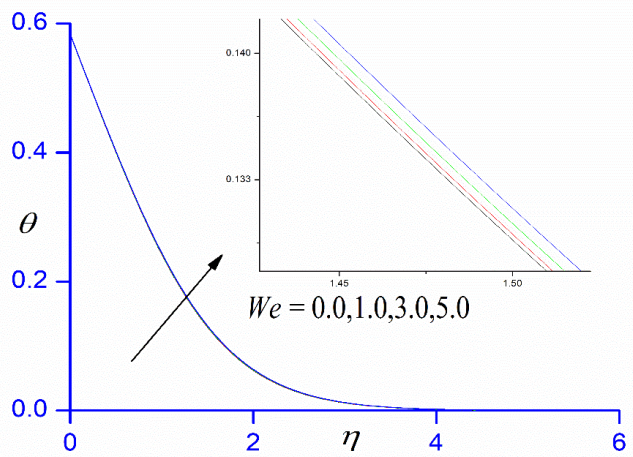

(b)

Fig. 3: Effect of the Weissenberg number We on $(a)$ the velocity and (b) temperature profiles, when $n=0.1, \operatorname{Pr}=7, M=1, S_{f}=$ $0.5, B i=0.3$ and $\xi-1$.

somewhat the temperature throughout the boundary layer regime.

Fig. 4 is plotted to highlight the effect of the magnetic parameter $M$ on the velocity and temperature distributions. From Fig. 4(a), it is observed that the velocity profile decreases with the rise in the values of the magnetic parameter $M$. The presence of a magnetic field in an electrically conducting fluid introduces a drag-like force known as Lorentz force, which acts against the flow, in the case where the magnetic field is applied vertically across the fluid flow. Hence, the magnetic resistive forces allow to slow down the fluid velocity. Moreover, it is found from Fig. 4(b) that the temperature is increased with the increase in the values of the magnetic parameter $M$. As a result, the momentum boundary layer thickness is reduced and the temperature throughout the boundary layer increases slightly with the increase in the magnetic parameter $M$. It is worth mentioning here that the resistance

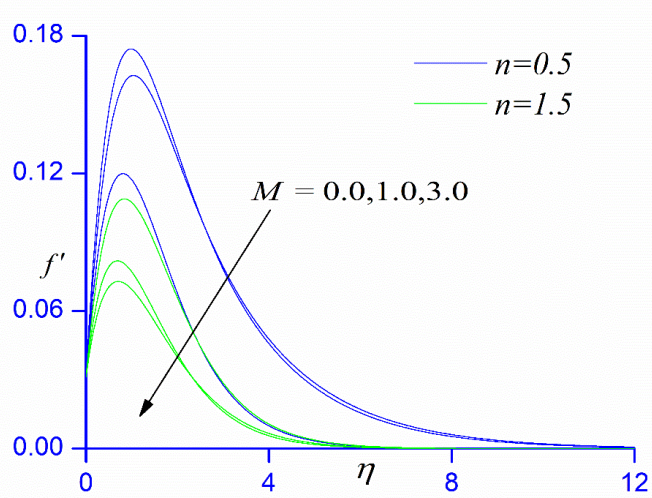

(a)

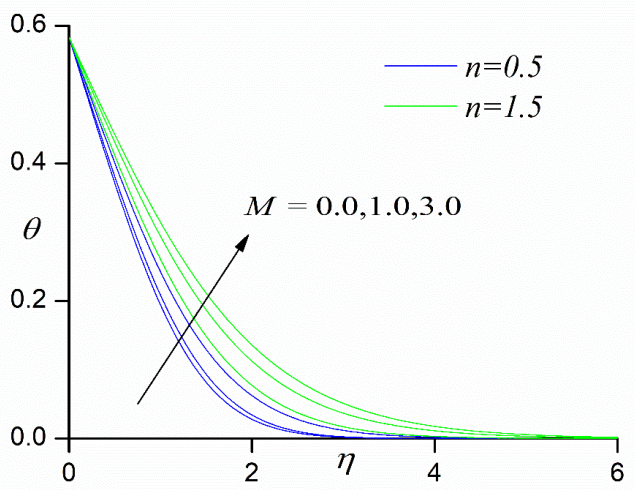

(b)

Fig. 4: Effect of the Magnetic parameter $M$ on (a) the velocity and (b) temperature profiles, when $n=0.5$ and $1.5, W e=0.3, P r=$ $7, S_{f}=0.5, B i=0.3$ and $\xi=1$.

caused by the presence of the external magnetic field will produce the heat in the system and increase the thermal boundary layer thickness.

Fig. 5 exhibits the influence of the effect of the velocity slip parameter $S_{f}$ on the velocity and temperature distributions. It is seen from Fig. 5(a) that the flow is markedly accelerated in the vicinity of the cone surface with the increase in the velocity slip parameter $S_{f}$. The velocity profiles plotted in Fig. 5(a) show the presence of peaks from some distance of the wall. These peaks are taken high values and shifted closer to the cone wall, when the value of the velocity slip parameter $S_{f}$ is increased. Thereafter, the momentum slip induces a notable acceleration in the flow near to the cone surface and decreases the momentum boundary layer thickness. On the contrary, it is observed that the momentum slip retards the fluid motion near and at the infinity boundary condition. Moreover, 


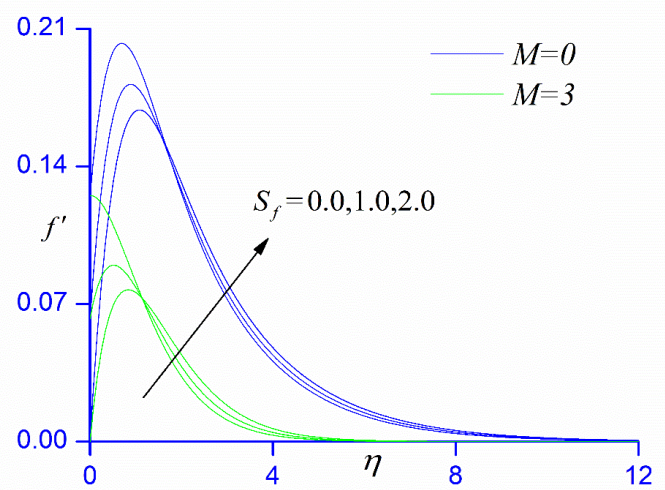

(a)

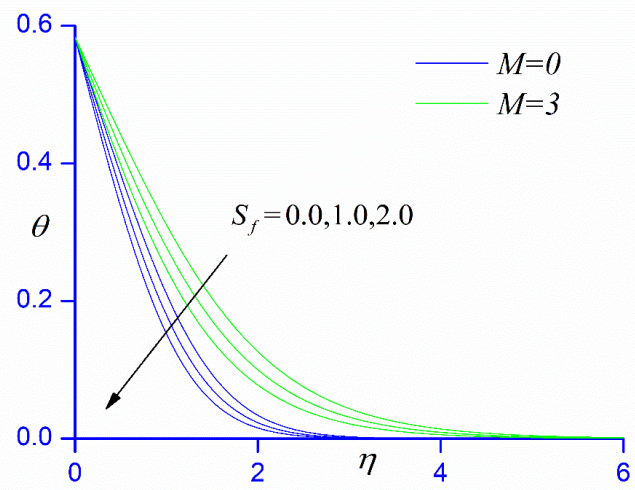

(b)

Fig. 5: Effect of the velocity slip parameter $S_{f}$ on $(a)$ the velocity and (b) temperature profiles, when $n=0.1, W e=0.3, \operatorname{Pr}=7, M=0$ and $3, B i=0.3$ and $\xi=1$.

dragging of the fluid adjacent to the cone surface is partially transmitted into the fluid, which induces a deceleration near the wall. However, this observation is eliminated and reversed further from the cone surface. From Fig. 5(b) , it is found that the temperature profiles decay monotonically from a maximum at the cone surface to the free stream, so that these profiles is converged at a large value of transverse coordinate $\eta$ to the zero value, again showing that a sufficiently large infinity boundary condition has been taken into account during the numerical computations. With greater momentum slip, the temperature in the boundary layer is substantially decreased, and hence the thermal boundary layer thickness is dropped appreciably with the increase in the velocity slip parameter $S_{f}$. Therefore, the regime is hottest when the momentum slip is absent and coolest with a strong hydrodynamic wall slip.

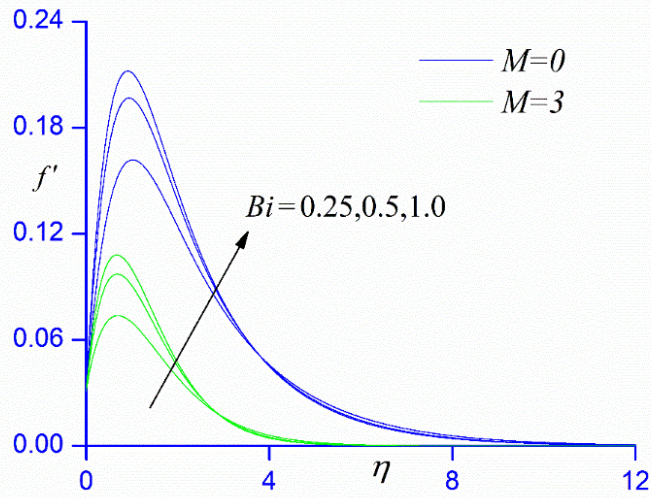

(a)

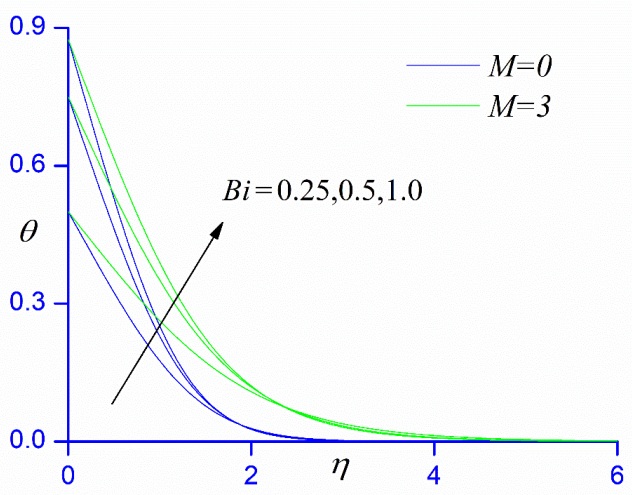

(b)

Fig. 6: Effect of the Biot number $B i$ on $(a)$ the velocity and $(b)$ temperature profiles, when $n=0.1, W e=0.3, P r=7, M=0$ and 3, $S_{f}=0.5$ and $\xi=1$.

Fig. 6 shows the influence of the Biot numberBion the velocity and temperature distributions. From Figs. 6(a) and Fig. 6(b), it is clearly noticed that both the velocity and the temperature are consistently increased with the increase in the Biot numberBiin the vicinity of the cone surface, so that the temperature becomes strongly enhanced at the cone surface. Hence, an increase in the value of the Biot number $B i$ allows to accelerate the flow and cool the boundary layer. It is also found that the momentum and thermal boundary layer thicknesses arise slightly when the Biot number $B i$ is increased. Physically, an increase in the Biot number $B i$ renders the fluid flow within the boundary layer progressively less sensitive to the heating effects at the cone surface, so that a increased quantity of thermal energy is transferred from the hot surface to the fluid, producing a fall in the temperature throughout the boundary layer. These results have important implica- 


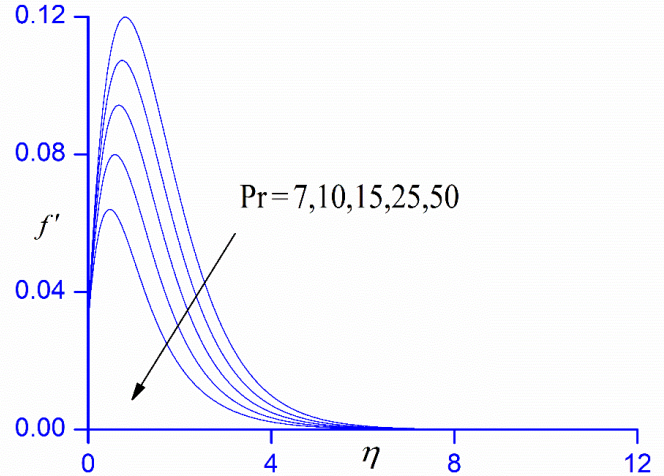

(a)

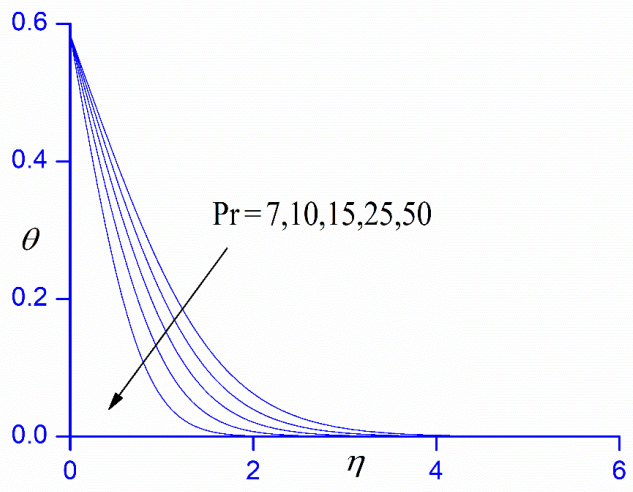

(b)

Fig. 7: Effect of the Prandtl number $\operatorname{Pr}$ on $(a)$ the velocity and $(b)$ temperature profiles, when $n=0.1, W e=0.3, M=1, S_{f}=$ $0.5, B i=0.3$ and $\xi=1$.

tions in the thermal polymer enrobing, since the Newtonian heating modifies the heat transferred to the polymer material, which in turn alters characteristics of the final product.

Fig. 7 represents the velocity and temperature profiles for various values of the Prandtl number $\mathrm{Pr}$.From Figs. 7(a) and Fig. 7(b) , it is found that an increase in the Prandtl number $\operatorname{Pr}$ decreases both the polymer flow velocity and the temperature throughout the boundary layer. Physically, the Prandtl number Pr represents the ratio of the viscosity to the thermal diffusivity. Hence, the rise in the values of the Prandtl number $P r$ will cause a large increase in the fluid viscosity, so that the fluid becomes extremely thick, and hence moves so slowly. The most prominent variation in the velocity and temperature distributions arises at intermediate distances from the cone surface. Furthermore, it is quite clear that the momentum and thermal boundary layers are thinner at large values of the Prandtl number $\mathrm{Pr}$ compared with the case where the values of the Prandtl number $P r$ are taken smaller. This fact is due to the inverse relation between the Prandtl numberPr and the thermal diffusivity. So, an increase in the Prandtl number $P r$ prevents spreading a large amount of heat within the boundary layer.

\section{Conclusions}

A mathematical model has been developed for the buoyancy driven MHD boundary layer flow of a Carreau fluid from a vertical cone. The transformed conservation equations have been solved with prescribed boundary conditions using the finite difference implicit KellerBox method, which has a second-order accuracy. Excellent convergence and stability characteristics are demonstrated by the Keller-Box scheme, which is capable of solving very strongly nonlinear rheological problems. The present simulations have shown that:

1. An increase in either the Carreau viscoelastic fluid parameter $W e$, the magnetic parameter $M$, the Convective heating $B i$, the Prandtl number $P r$, the power law index $n$ or the steam wise coordinate $\xi$ allows to decrease the fluid velocity near the cone surface.

2. The presence of the velocity slip condition $S_{f}$ allows to increase the fluid velocity near the cone surface.

3. An increase in either the Carreau viscoelastic fluid parameter $W e$, the magnetic parameter $M$, the power law index $n$ or the steam wise coordinate $\xi$ allows to improve the temperature throughout the boundary layer.

4. The temperature distribution throughout the boundary layer can be reduced by the increase in either the velocity slip parameter $S_{f}$, the Convective heating $B i$ or the Prandtl number Pr.

5. The effect of viscous friction at the cone surface can be minimized by increasing the values of the magnetic parameter $M$, the velocity slip parameter $S_{f}$ and the Convective heating $B i$.

6. The local heat transfer rate is enhanced with the increase in the velocity slip parameter $S_{f}$, while an inverse result is observed when the parameters $M$ and $B i$ are increased.

The current study has explored an interesting viscoelastic model for smart coating transport phenomena. 
which are currently of interest in polymer coating applications. Time-dependent effects have been neglected. Future studies will therefore address transient enrobing viscoelastic polymer fluid transport phenomena for alternative geometries (Cylinder, Wedges and Plates).

Acknowledgement: The authors appreciate the constructive comments of the reviewers which led to definite improvement in the paper. The corresponding author $\mathrm{CH}$. Amanulla is thankful to Mohammed Rafeek Mysore, IT analyst in Tata Consultancy Services (TCS) Miami FL, USA, for his financial support.

\section{Nomenclature}

\begin{tabular}{|c|c|}
\hline \multicolumn{2}{|c|}{ List of symbols } \\
\hline$A$ & Half angle of the cone, $m$ \\
\hline $\mathrm{B}_{0}$ & Externally imposed radial magnetic field \\
\hline$c$ & Specific heat , $J k^{-1} K^{-1}$ \\
\hline$C_{f}$ & Skin friction coefficient \\
\hline$f$ & Dimensionless stream function \\
\hline$G r$ & Grashof number \\
\hline g & Gravity field \\
\hline$k$ & Thermal conductivity of fluid, $W m^{-1} K^{-1}$ \\
\hline$n$ & Power law index parameter \\
\hline$K_{0}$ & Thermal jump factor , $m^{-1}$ \\
\hline$N_{0}$ & Momentum slip factor , $m^{-1}$ \\
\hline $\mathrm{Nu}$ & Local Nusselt number \\
\hline$M$ & Magnetic parameter \\
\hline$P$ & Pressure, $\mathrm{Pa}$ \\
\hline $\operatorname{Pr}$ & Prandtl number $(\operatorname{Pr}=v / \alpha)$ \\
\hline$S_{f}$ & Velocity slip parameter \\
\hline$B i$ & Biot number \\
\hline$T$ & Temperature, $K$ \\
\hline$U, V$ & Dimensionless velocity components, $m s^{-1}$ \\
\hline$W e$ & Weissenberg number \\
\hline$x$ & Stream wise coordinate, $m$ \\
\hline$y$ & Transverse coordinate, $m$ \\
\hline
\end{tabular}

\section{Greek symbols}

$\alpha \quad$ Thermal diffusivity, $m^{2} s^{-1}$

$\beta \quad$ Thermal expansion coefficient,$K^{-1}$

$\eta \quad$ Dimensionless transverse coordinate

$\theta \quad$ Dimensionless temperature

$\mu_{0} \quad$ Zero shear rate viscosity, $P$ as

$v \quad$ Kinematic viscosity $\left(v=\mu_{0} / \rho\right), m^{2} s^{-1}$

$\xi \quad$ Dimensionless steam wise coordinate

$\rho \quad$ Fluid density, $\mathrm{Kgm}^{-3}$

$\sigma \quad$ Electrical conductivity, $\Omega^{-1} m^{-1}$

$\tau_{w} \quad$ Wall shear stress

$\psi \quad$ Stream function, $m^{2} s^{-1}$

$\Gamma \quad$ Time-dependent material constant, $s$

$\nabla \quad$ Nabla operator

Subscripts

$w \quad$ Wall condition

$\infty \quad$ Free stream condition

\section{References}

[1] Landau, L. D. and Levich, B., “Dragging of liquid by a plate,” Acta Physiochim. USSR, 1942, 17, 42-54.

[2] Lawrence, C. J. and Zhou, W., "Spin coating of non-Newtonian fluids," Journal of Non-Newtonian Fluid Mechanics, 1991, 39, 137-187.

[3] Samson, J. A. and Spencer, B. S., "Coating flow of nonNewtonian fluids on a flat rotating disk," Industrial \& Engineering Chemistry Research, 1984, 23(4), 432-436.

[4] Osvaldo, C. H., Jorge, L. G. and Ramón, L. C., "Viscous flow on the outside of a horizontal rotating cylinder-II. Dip coating with a non-Newtonian fluid," Chemical Engineering Science, 1986, 41(11), 2707-2713.

[5] Zevallos, G. A., Carvalhoa, M. S. and Pasquali, M., "Forward roll coating flows of viscoelastic liquids," Journal of NonNewtonian Fluid Mechanics, 2005, 130, 96-109.

[6] Mitsoulis, E., "Fluid flow and heat transfer in wire coating: A review," Advances in Polymer Technology, 1986, 6, 467-487.

[7] Mark, J. E., "Physical Properties of Polymers Handbook," AIP Press, Woodbury, New York, USA. 1996.

[8] Uddin, M. J., Khan, W. A. and Ismail, A. I. Md., "Free Convective Flow of Non-Newtonian Nanofluids in Porous Media with Gyrotactic Microorganism," J. Therm. Heat Tran., 2013, 27(2), 326-333.

[9] Uddin, M. J., Bég, O. A. and Amin, N., "Hydromagnetic transport phenomena from a stretching or shrinking nonlinear nanomaterial sheet with Navier slip and convective heating: A model for bio-nano-materials processing,"J. Mag. Mag. Mat., 2014, 368, 252-261. http://dx.doi.org/10.1016/j.jmmm.2014.05.041

[10] Hayat, T., Farooq, S. and Alsaedi, A., "Mixed convection peristaltic motion of Copper-water nanomaterial with velocity slip effects in a curved channel," Computer Methods and Programs in Biomedicine, 2017, 142, 117-128. https://doi.org/10.1016/j.cmpb.2017.02.006

[11] Shahid, F., Hayat, T., Ahmed, A. and Bashir, A., "Numerically framing the features of second order velocity slip in mixed convective flow of Sisko nanomaterial considering gyrotactic microorganisms," Int. J. Heat. Mass. Trans., 2017, 112, 521-532.

[12] Uddin, M. J., Yasser, A., Bég, O. A. and Kabir, M. N., “Numerical solutions for gyrotactic bioconvection in nanofluid-saturated porous media with Stefan blowing and multiple slip effects," Computers \& Mathematics with Applications, 2016, 72 (10), 2562-2581. http://dx.doi.org/10.1016/j.camwa.2016.09.018

[13] Amanulla, C.H., Nagendra, N., and Surya Narayana Reddy, M., "Numerical Study of Thermal and Momentum Slip Effects on MHD Williamson Nanofluid from an Isothermal Sphere", Journal of Nanofluids, 2017, 6(6), 1111-1126. https://doi.org/10.1166/jon.2017.1405

[14] Nagendra, N., Amanulla, C.H., and Surya Narayana Reddy, M., "Mathematical Analysis of Non-Newtonian Nanofluid Transport Phenomena Past a Truncated Cone with Newtonian Heating", Journal of Naval Architecture and Marine Engineering, 2018, 15(1), 17-35. http://dx.doi.org/10.3329/jname.v15i1.29966

[15] Tripathi, D. and Bég, O. A., “Mathematical modelling of heat transfer effects on swallowing dynamics of viscoelastic flood 
bolus through the human esophagus," International Journal of Thermal Sciences, 2013, 70, 41-53.

[16] Cheng, C-Y., "Natural convection heat transfer about a vertical cone embedded in a tridisperse porous media with constant wall temperature," Transport in porous media, 2015, 107, 765779.

[17] Yih, K.A., "Effect of radiation on natural convection about a truncated cone," International Journal of Heat and Mass Transfer, 1999, 42, 4299-4305.

[18] Gorla, R. S. R., Schoren, W. R. and Takhar, H. S., "Natural convection boundary layer flow of a micropolar fluid over an isothermal cone," Acta Mechanica, 1986, 61, 139-152.

[19] Uddin, M. J., Bég, O. A. and Uddin, M.N., "Energy conversion under conjugate conduction, magneto-convection, diffusion and nonlinear radiation over a non-linearly stretching sheet with slip and multiple convective boundary conditions," Energy, 2016, 115, 1119-1129. http://dx.doi.org/10.1016/j.energy.2016.05.063

[20] Shahid, F., Ahmed, A., Hayat, T. and Bashir A., "Peristaltic transport of Johnson-Segalman fluid with homogeneousheterogeneous reactions: a numerical analysis,"J. Braz. Mech. Sci. Eng., 2018, 40. https://doi.org/10.1007/s40430-018-1173$\mathrm{x}$

[21] Hayat, T., Farooq, S., Ahmad, B. and Alsaedi, A., "Characteristics of convective heat transfer in the MHD peristalsis of Carreau fluid with Joule heating," AIP Advances, 2016, 6. http://dx.doi.org/10.1063/1.4945767

[22] Hayat, T., Farooq, S., Ahmad, B. and Alsaedi, A., "Numerical analysis for radial MHD and mixed convection effects in peristalsis of non-Newtonian nanomaterial with zero mass flux conditions," Results in Physics, 2017, 7, 451-458.

[23] Saleem, S., Nadeem, S. and Haq, R. Ul., "Buoyancy and metallic particle effects on an unsteady water-based fluid flow along a vertically rotating cone," Eur. Phys. J., 2014, 129.

[24] Saleem, S., Nadeem, S. and Sandeep, N., "A Mathematical Analysis of Time Dependent Flow On a Rotating Cone in a Rheological Fluid," Propulsion and Power Research, 2017, 6, 233241.

[25] Amanulla, C.H., Nagendra, N., and Suryanarayana Reddy, M., "Computational analysis of non-Newtonian boundary layer flow of nanofluid past a semi-infinite vertical plate with partial slip", Nonlinear Engineering. 2018, 7(1), 29-43. https://doi.org/10.1515/nleng-2017-0055

[26] Saleem, S., Nadeem, S. and Awais, M., "Time dependent second order viscoelastic fluid flow on a rotating cone with Heat generation and chemical reaction," J. Aerospace Eng., 2016, 29(4).

[27] Saleem, S., AlQarni, M. M., Nadeem, S. and Sandeep, N., "Convective heat and mass transfer in magneto Jeffrey fluid flow on a rotating cone with heat source and chemical reaction," Comm. Theo. Phys., 2018.

[28] Amanulla, C.H., Nagendra, N., and Suryanarayana Reddy, M., "Thermal and Momentum Slip Effects on Hydromagnetic Convection Flow of a Williamson Fluid Past a Vertical Truncated Cone", Frontiers in Heat and Mass Transfer, 2017, 9, 22.

[29] Khellaf, K. and Lauriat, G., "Numerical study of heat transfer in a non-Newtonian Carreau-fluid between rotating concentric vertical cylinders," Journal of Non-Newtonian Fluid Mechanics, $2000,89,45-61$.
[30] Khan, M., Hussain, M. H. and Azam, M., "Magnetohydrodynamic flow of Carreau fluid over a convectively heated surface in the presence of non-linear radiation," Journal of Magnetism and Magnetic Materials, 2016, 412, 63-68.

[31] Raju, C. S. K. and Sandeep, N., "Falkner-Skan flow of a magnetic-Carreau fluid past a wedge in the presence of cross diffusion effects," The European Physical Journal Plus, 2016, 131.

[32] Akbar, N. S., Nadeem, S., Rizwan, H. and Shiwei, Ye., “MHD stagnation point flow of Carreau fluid toward a permeable shrinking sheet: Dual solutions," Ain Shams Engineering Journal., 2014, 5(4), 1233-1239.

[33] Reddy, M. G. and Sandeep, N., “Heat and mass transfer in radiative MHD Carreau fluid with cross diffusion," Ain Shams Engineering Journal, 2016. http://dx.doi.org/10.1016/j.asej.2016.06.012

[34] Black, W.B., "Wall slip and boundary effects in polymer shear flows", PhD Thesis, Chemical Engineering, University of Wisconsin - Madison, USA, 2000.

[35] Wang, S.-Q., Drda, P. A. and Inn, Y.-W., “Exploring molecular origins of sharkskin, partial slip, and slope change in flow curves of linear low density polyethylene," Journal of Rheology, 1996, 40(5), 875-898.

[36] Piau, J.-M., Kissi, N. E., Toussaint, F. and Mezghani, A., “Distortions of polymer extrudates and their elimination using slippery surfaces," Rheologica Acta, 1995, 34, 40-57.

[37] Piau, J. M. and Kissi, N. E., "Measurement and modelling of friction in polymer melts during macroscopic slip at the wall," Journal of Non-Newtonian Fluid Mechanics, 1994, 54, 121-142.

[38] Lim, F. J. and Schowalter, W. R., "Wall slip of narrow molecular weight distribution polybutadienes," Journal of Rheology, 1989, 33(8), 1359-1382.

[39] Hatzikiriakos, S. G. and Kalogerakis, N., "A dynamic slip velocity model for molten polymers based on a network kinetic theory," Rheologica Acta, 1994, 33, 38-47.

[40] Liu, Y. and Gehde, M., "Effects of surface roughness and processing parameters on heat transfer coefficient between polymer and cavity wall during injection molding," The International Journal of Advanced Manufacturing Technology., 2016, 84, 1325-1333.

[41] Hatzikiriakos, S. G. and Mitsoulis, E., "Slip effects in tapered dies," Polymer Engineering \& Science, 2009, 49(10), 19601969.

[42] Crane LJ, Flow past a stretching plate. Z Angew Math Phys 1970, 21, 645-647.

[43] Grubka LJ, Bobba KM, Heat transfer characteristics of a continuous, stretching surface with variable temperature. Heat Trans-T ASME 1985, 107, 248-250.

[44] Wang CY, Free convection on a vertical stretching surface. ZAMM 1989, 69, 418-420.

[45] Gupta PS, Gupta AS, Heat and mass transfer on a stretching sheet with suction or blowing. Can J Chem Eng 1977, 55, 744746.

[46] Amanulla, C. H., Nagendra, N. and Suryanarayana Reddy, M., "Numerical Simulations on Magnetohydrodynamic NonNewtonian Nanofluid Flow Over a Semi-Infinite Vertical Surface with Slip effect," Journal of Nanofluids, 2018, 7(4), 718730. https://doi.org/10.1166/jon.2018.1499

[47] Amanulla, C. H., Nagendra, N. and Suryanarayana Reddy, M., "Numerical Simulation of Slip Influence on the Flow of a MHD 
Williamson Fluid Over a Vertical Convective Surface," Nonlinear Engineering, 2018, 1-13. https://doi.org/10.1515/nleng2017-0079

[48] Amanulla, C.H., Nagendra, N. and Suryanarayana Reddy, M., "Multiple Slip Effects on MHD and Heat Transfer in a Jeffery Fluid over an Inclined Vertical Plate," Int. J. Pure. App. Math., 2017, 113(7), 137-145.

[49] Merkin, J. H., "Natural convection boundary layer flow on a vertical surface with Newtonian heating," International Journal of Heat and Fluid Flow, 1994, 15, 392-398.

[50] Ramzan, M., Farooq, M., Alhothuali, M. S., Malaikah, H. M., Cui, W. and Hayat, T., "Three dimensional flow of an Oldroyd-B fluid with Newtonian heating," Int. J. Num. Meth. Heat \& Fluid Flow, 2015, 25, 68-85.

[51] Muhammad, A., Hayat, T., Abdul, Q. and Ahmed, A., "Newtonian heating in a flow of thixotropic fluid," European Phys. J., 2013, 128.

[52] Uddin, M. J., Bég, O. A., Amran, N. and Ismail, A. I. MD., "Lie group analysis and numerical solutions for magnetoconvective slip flow of a nanofluid over a moving plate with a Newtonian heating boundary condition," Canadian J. Physics, 2015, 93, 1-10.

[53] Alam, M.M., Alim, M.A., and Chowdhury, M.M.K., "Free Convection from a Vertical Permeable Circular Cone with Pressure Work and Non-Uniform Surface Temperature," Nonlinear Analysis: Modelling and Control, 2007, 12(1), 21-32.
[54] Cebeci, T. and Bradshaw, P., "Physical and Computational Aspects of Convective Heat Transfer," Springer, New York, 1984.

[55] Keller, H. B., "A new difference method for parabolic problems," J. Bramble (Editor), Numerical Methods for Partial Differential Equations, Academic Press, New York, USA, 1970.

[56] Amanulla, C. H., Nagendra, N. and Suryanarayana Reddy, M., "MHD Flow and Heat Transfer in a Williamson Fluid from a Vertical Permeable Cone with Thermal and Momentum Slip Effects: A Mathematical Study," Frontiers in Heat and Mass Transfer, 2017, 8, 40. http://dx.doi.org/10.5098/hmt.8.40

[57] Amanulla, C. H., Nagendra, N., Rao A. S, Bég O. A. and Kadir A., "Numerical Exploration of Thermal Radiation and Biot Number Effects on the Flow of a Non-Newtonian MHD Williamson Fluid over a Vertical Convective Surface," Heat Transfer-Asian Research, 2018, 47(2), 286-304. https://doi.org/10.1002/htj.21303

[58] Nagendra, N., Amanulla, C. H., Sudhakar Reddy, M. and Prasad, V. R., "Hydromagnetic Flow of Heat and Mass Transfer in a Nano Williamson Fluid Past a Vertical Plate with Thermal and Momentum Slip Effects: Numerical Study," Nonlinear Engineering, 2018, 1-18.

[59] Nagendra, N., Amanulla, C. H. and Sudhakar Reddy, M., "Slip Effects on MHD Flow of a Williamson Fluid from an Isothermal Sphere: A Numerical Study," Modelling, Measurement and Control B, 2017, 86(3), 782-80. 